\title{
The New FundamentalWave Library for Modeling Rotating Electrical Three Phase Machines
}

\author{
Christian Kral Anton Haumer \\ AIT Austrian Institute of Technology $\mathrm{GmbH}$ \\ Mobility Department, Electric Drive Technologies \\ Giefinggasse 2, 1210 Vienna, Austria
}

\begin{abstract}
This paper introduces the new FundemantalWave library which is included in the Modelica Standard Library 3.2. The presented Modelica package provides models and components of rotating electrical three phase machines. The presented electrical machine models are fully compatible with the original Machines library of the electrical domain but rely on the concept of the magnetic potential and magnetic flux fundamental waves. In this article, the connector concept, the components and electric machine models of the FundemantalWave package will be explained. Additionally, the didactic advantages and the flexibility of the proposed package in with respect to considering more enhanced and sophisticated effects will be discussed.
\end{abstract}

Keywords: Rotating electrical three phase machines, fundamental wave, time transients, Modelica Standard Library MSL

\section{Introduction}

Electric machines models have been introduced to the Modelica Standard Library (MSL) in 2004. Since then the package has continuously been enhanced and additional machine types and effects have been added to the Modelica.Electrical. Machines library (short Machines library). More sophisticated loss effects and a consistent thermal concept have been implemented for the library version included in the MSL 3.2. . The more sophisticated loss models include

- friction losses,

- eddy current core losses,

- stray load losses and
- brush losses,

and have been published in [1]. These loss models have been incorporated in the Machines library such way that they can be applied for both the original Machines and the new Modelica.Magnetic.FundamentalWave library. The main difference between the two machines packages is that Machines uses current, voltage and flux linkage space phasors (vectors) [2-5] whereas FundamentalWave applies complex vectors for physical representation of the the magnetic flux and the magnetic potential difference.

The motivation for introducing a new package for rotating electrical three phase machine was the need for modeling different effects based on physical magnetic quantities and models. An example for modeling global demagnetization effects of the permanent magnets in synchronous machines is presented in [6]. Additonal applications will be discussed in section 5 .

An original implementation of an electric machines library based on magnetic potential differences and magnetic flux vectors has been presented by Beuschel [7]. In this paper, however, DC and three phase electrical machine have been modeled based on a magnetic connector which is designed slightly different from the one introduced in the Fundamentalwave library. In the original implementation of Beuschel the objective was the universal applicability of the proposed concept to different types of machines. The main focus of the Fundamental Wave library was motivated by a clear physical interpretation of the quantities in the connector such that the library can be applied to model complex physical phenomena in electrical machines.

The Fundamentalwave library models rely on the following assumptions:

- only symmetrical three phase induction machines are modeled 
- in the current implementation only linear magnetic circuit elements are taken into account

- deep bar effects and higher harmonic spatial electro magnetic wave effects are also not considered

\section{Concept and Magnetic Connector}

In the package Modelica.Magnetic.FluxTubes the concept of magnetic ports has been introduced to model one dimensional flux tubes. The generic magnetic port of FluxTubes,

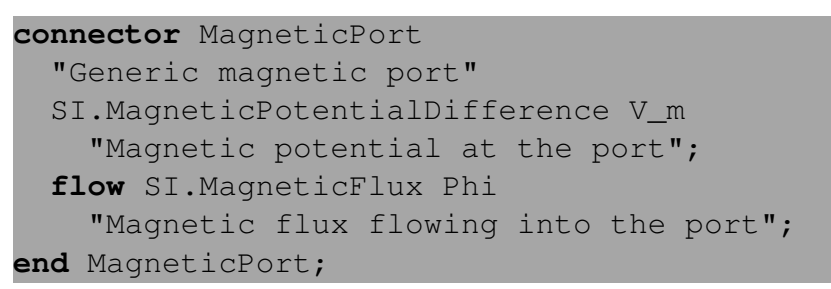

consists of the magnetic potential difference as potential variable and the magnetic flux as flow variable.

In a radial field electrical machine usually only the fundamental field components is modeled. As an example, the two dimensional field distribution of a four pole asynchronous induction machine is depicted in Fig. 1. The magnetic field quantities in the air gap, e.g., the magnetic potential difference, can be approximated by a fundamental wave with respect to one pole pair, as it is presented in Fig. 2. In this figure the angle $\varphi$ refers to one pole pair $(\varphi=2 \pi)$ of the machine. The angle $\varphi$ is also referred to as electrical angle. The magnitude and the phase shift of the fundamental wave in Fig. 2 can be represented by a phasor in the complex plane as depicted in Fig. 3. In the FundamentalWave library the complex magnetic potential difference and the complex magnetic flux are represented by a real and imaginary part, respectively:

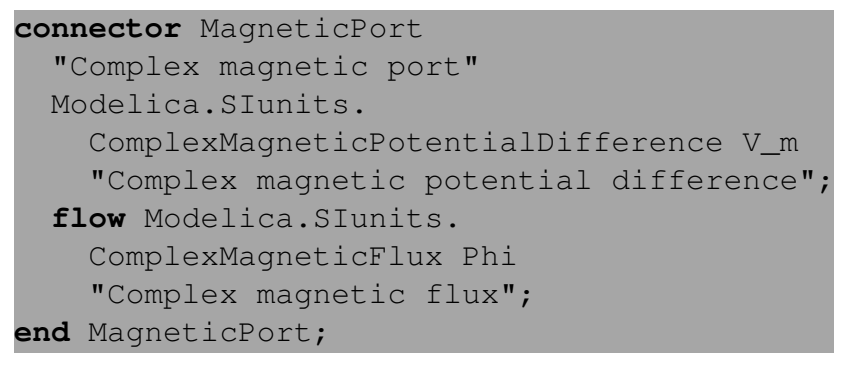

The relationship between the real and imaginary parts of the phasors and the spatial waveform of the connector components is given by:

$$
\begin{aligned}
V_{m}(\varphi) & =\operatorname{Re}\left[\left(V_{m, \mathrm{re}}+j V_{m, \mathrm{im}}\right) e^{-j \varphi}\right] \\
& =V_{m, \mathrm{re}} \cos (\varphi)+V_{m, \mathrm{im}} \sin (\varphi) \\
\Phi(\varphi) & =\Phi_{\mathrm{re}} \cos (\varphi)+\Phi_{\mathrm{im}} \sin (\varphi)
\end{aligned}
$$

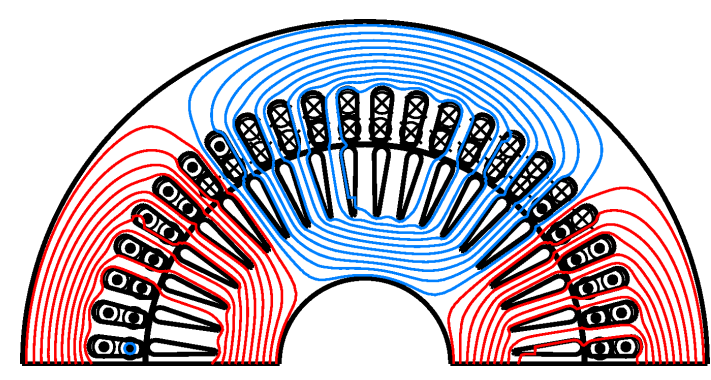

Figure 1: Magnetic field distribution of and asynchronous induction machine at no load operation

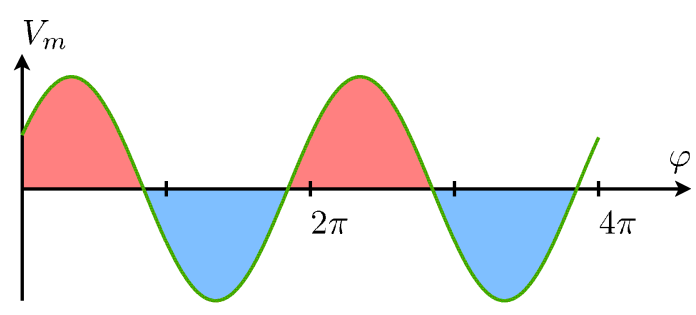

Figure 2: Fundamental wave of the magnetic potential difference

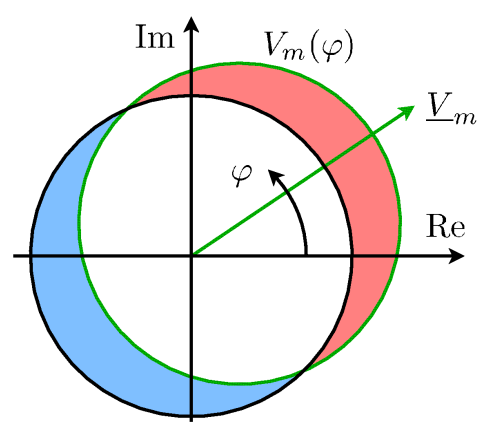

Figure 3: Complex phasor of the magnetic potential difference

It is important to note that the magnetic potential difference of the connector definition refers to the total magnetic potential difference excited by all poles of the machine.

In both the Machines and the new FundamentalWave library only spatial fundamental wave effects of spacial magnetic quantities are considered. Higher harmonic spatial wave effects due to

- the spatial reluctance distribution of the slots,

- the magneto motive force (magnetic potential difference) harmonics due to the current of the coils embedded in slots, and,

- if present, the field distribution of the permanent magnets, 


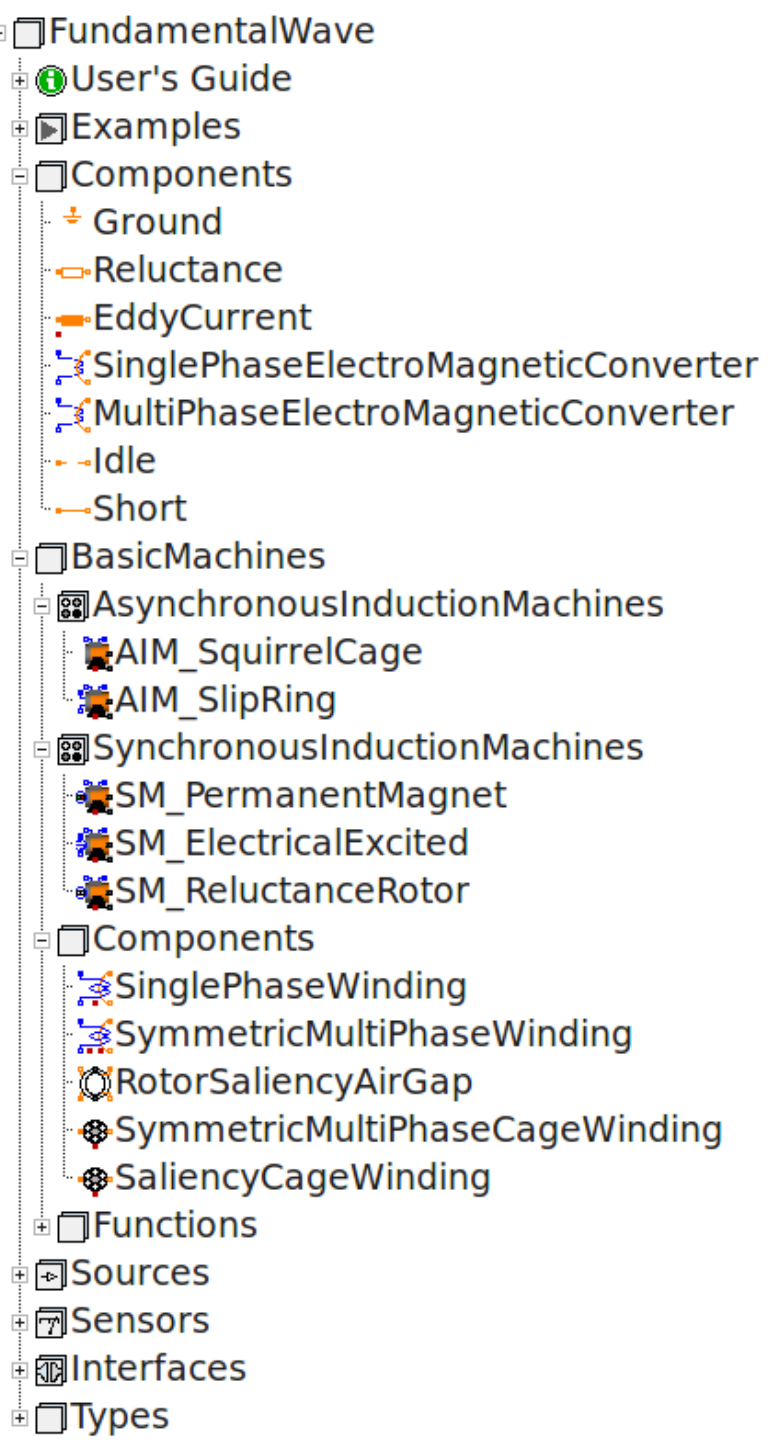

Figure 4: Structure of the Fundamentalwave library

are not taken into account. The impact of higher harmonic time harmonics in the currents and voltages is, however, fully taken into account by the spatial fundamental wave representation.

\section{Components}

An overview of the packages and components included in the Fundamentalwave library is presented in Fig. 4. In this chapter the generic components, the machine specific components and the background of the thermal super connector will be presented. Chapter 4 will then go into detail with the topology specific machine models.

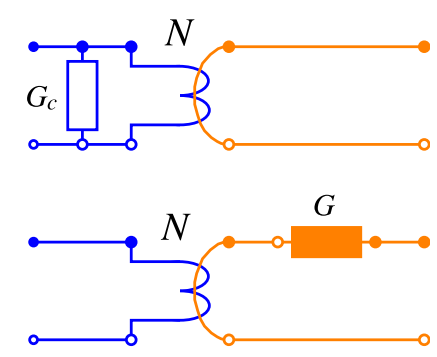

Figure 5: Equivalent consideration of eddy current losses in a multi phase electrical and a fundamental wave magnetic circuit

\subsection{Generic FundamentalWave Components}

The magnetic Ground model sets the real and imaginary part of the magnetic potential difference of the connector to zero. The complex flux components are not affected in this model.

In the Reluct ance model a linear relationship between the magnetic potential difference and the magnetic flux components is considered. In order to take magnetic saliences of the reluctance into account a new saliency type is defined, which consists of a d and $q$ components. The equations of the reluctance model are:

$(\mathrm{pi} / 2) \star V \_m \cdot r e=R \_m \cdot d * P h i \cdot r e ;$
$(p i / 2) \star V \_m \cdot i m=R \_m \cdot q * P h i \cdot i m ;$

In these equations the term $\mathrm{pi} / 2$ results of the averaging of the sinusoidal waveform of the magnetic flux density over one pole pair. In a fully symmetrical magnetic circuit the $\mathrm{d}$ and $\mathrm{q}$ component of the reluctance parameter $R \_m$ are equal. Saliency effects with unequal $\mathrm{d}$ and $\mathrm{q}$ component of the reluctance have to be applied with care, since the saliency model always refers to the specific reference frame of the connectors.

The Eddycurrent loss model is designed in the style of the Fluxtubes package:

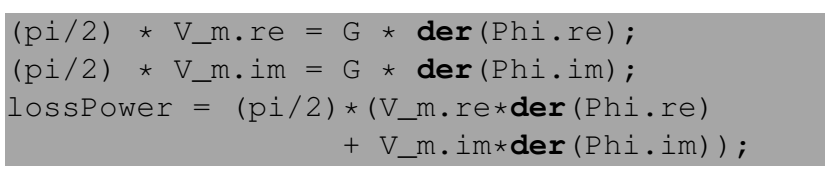

In an electric machine the eddy current loss model can usually also be represented in an electrical equivalent circuit as depicted in Fig. 5. The equivalent representation of the two circuits, however, relies on a symmetrical electro magnetic coupling as it is explained in the following paragraph. For an $m$ phase electrical circuit the parameter relationship

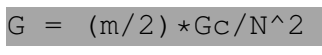

applies. 


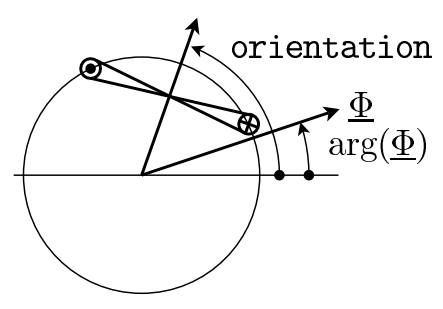

Figure 6: Orientation of a single phase coil and complex magnetic flux phasor

The SinglePhaseElectroMagneticConverter considers Ampere's law and the induction law for a single phase coil. Model parameters are the effective number of turns and the orientation, both with respect to the fundamental wave:

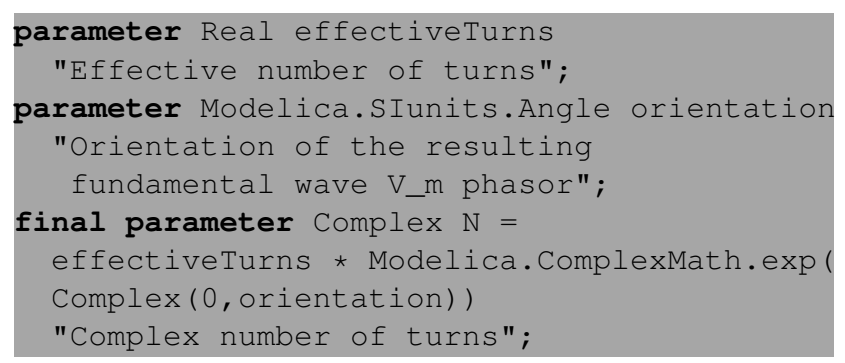

Such a single phase coil is depicted in Fig. 6 and the relevant model equations are:

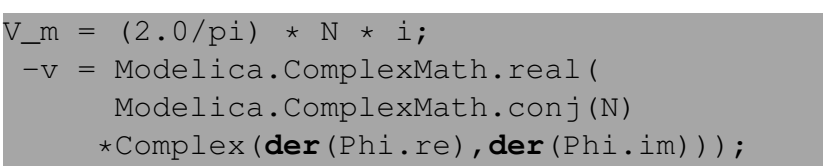

In the MultiPhaseElectroMagneticConverter, incorporating $m$ phases, an array of $m$ single phase electro magnetic converters is instantiated and connected with the multi phase electrical connectors. The magnetic ports of the single phase electro magnetic converters are series connected, since the same flux applies to each coupling element.

\subsection{Machine Specific Models}

For modeling electric machines some more specific models are provided. These models are single and symmetrical multi phase windings including

- winding resistance including thermal connector,

- leakage inductance and field, respectively,

- zero inductance,

- core losses associated with the respective winding including thermal connector, and the

- electro magnetic converter.

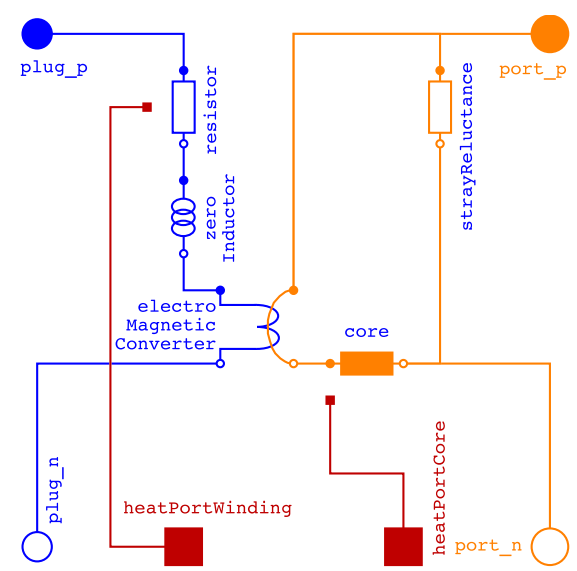

Figure 7: Symmetrical multi phase winding

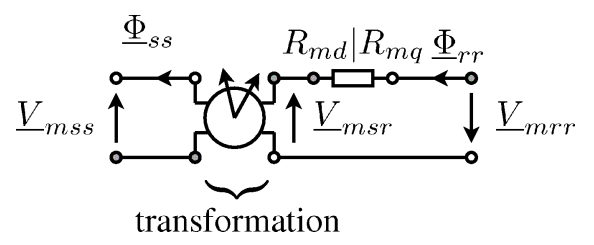

Figure 8: Stator and rotor fixed complex fluxes and magnetic potential differences of the air gap model

Additional machine specific models are the air gap model (with rotor saliency) as well as the symmetrical and salient rotor cage windings.

The symmetricalMultiPhaseWinding model is depicted in Fig. 7. The stray field and the core losses of the winding are considered in the magnetic domain. Since the modeled stray field implies and an ideal coupling of the $m$ electrical phases, the zero inductance of the machine has to be considered separately [8]. The thermal connectors of the winding resistor and core loss model are externally available.

In the RotorSaliencyAirgap model different physical effects are taken into account. First, the stator and rotor magnetic ports have different fundamental wave rotational frequencies as they refer to different reference frames, see Fig. 8:

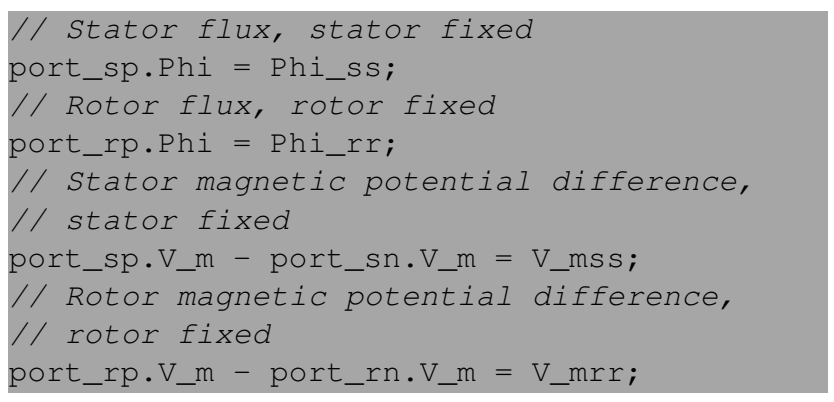

The quantities that either refer to the stator or rotor reference frame are transformed by means of a rotator that is derived from the electrical angular difference 
gamma between mechanical stator and rotor flangethus multiplied with the number of pole pairs $\mathrm{p}$ :

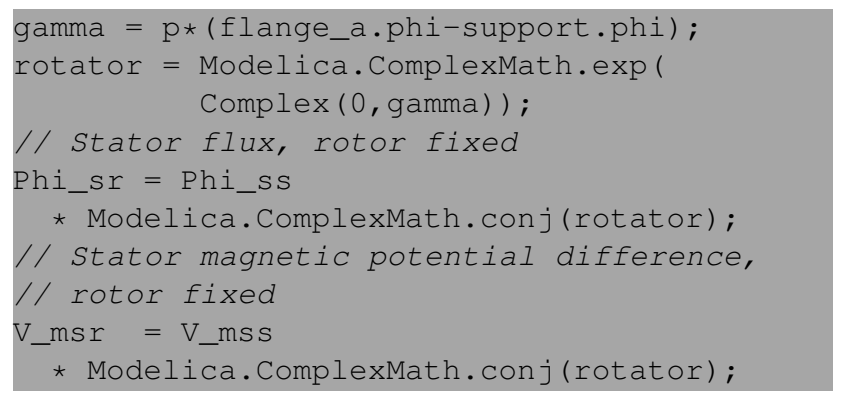

Second, the magnetic reluctance of the air gap due to rotor saliency is taken into account, since the relationships between the components of the fluxes and the magnetic potential differences are considered with respect to the rotor reference frame.

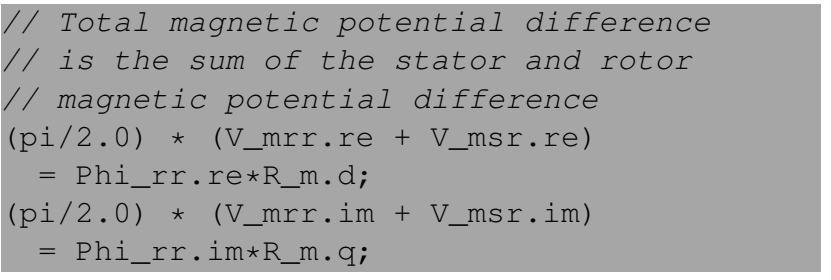

Cross coupling effects are not considered in this model.

All the electric machine models provided by the Fundamentalwave library do not take the reluctances of the stator and rotor teeth and yoke into account. Therefore, the total main field reluctance of the machine is seen as an equivalent air gap reluctance. Third, the inner torque of the air gap is calculated:

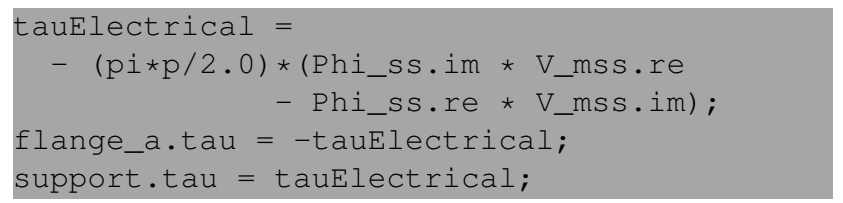

The symmetricMultiphaseCagewinding and SaliencycageWinding are designed in a similar way as the symmetric winding models. The main difference is the inherent short circuit of the cage winding and thus the cage models have only magnetic and thermal connectors, respectively. An example of a symmetric rotor cage model is depicted in Fig. 9. In this implementation the rotor cage is modeled as an equivalent three phase winding - which is already implied by the machine parameters rotor resistance $\mathrm{Rr}$ and rotor leakage inductance Lrsigma, which both refer to an equivalent stator winding. Rotor core losses are currently not considered in the cage winding models.

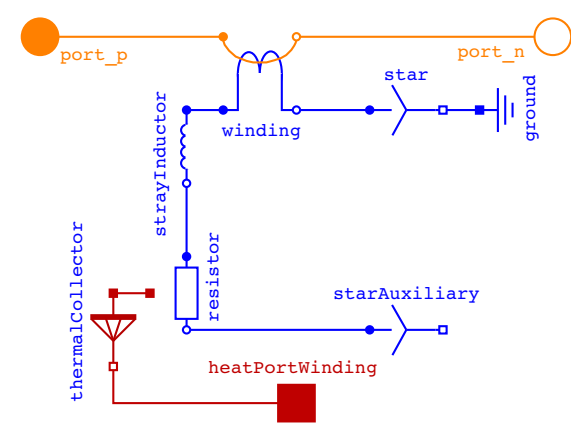

Figure 9: Symmetric rotor cage model

\subsection{Thermal Super Connectors}

For all the electric machine models thermal super connector are introduced. The super connectors are only implemented in the Machines library, since both the Machines and the FundamentalWave library use the same connector definitions. The super connector contains instances of thermal connectors. Each thermal connector is associated with the actual temperature and heat flow of one particular loss effect. Since the different machine models have different topologies, different super connector definitions are used. The common heat ports of all induction machine models are summarized in the partial model connector:

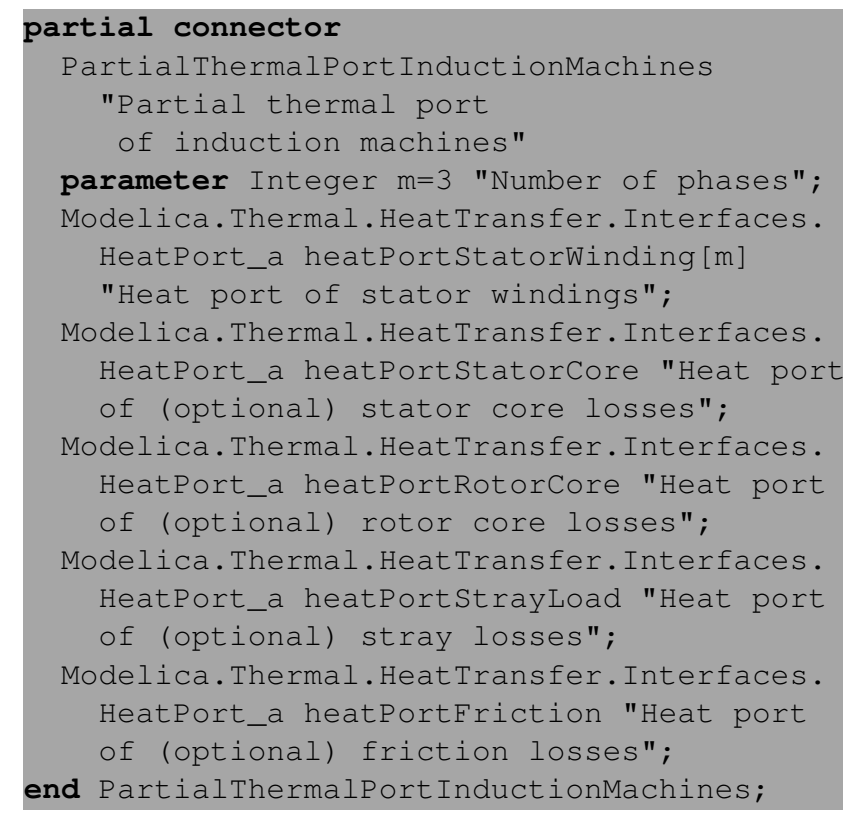

The basic thermal super heat port contains a connector for the stator winding (copper losses), the stator core (currently only stator eddy current losses), the rotor core (currently not utilized), stray load losses and the friction losses. Each connector used for the particular induction machine models extends from this partial connector definition and adds machine specific parameters and heat ports. For example, the thermal super 
connector definition for permanent magnet induction machines is:

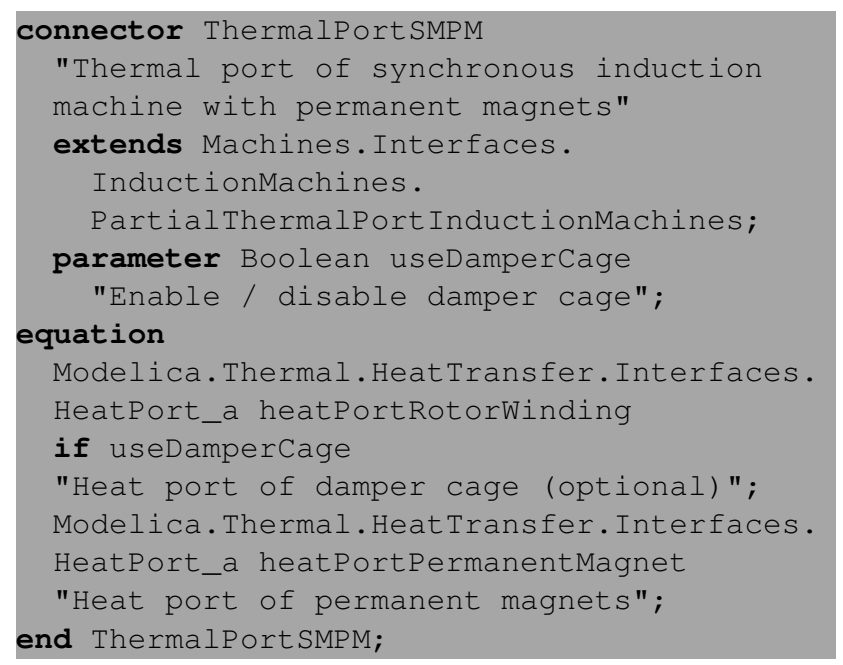

Since the permanent magnet synchronous machines are implemented with an optional damper cage, the boolean parameter useDamperCage has to be utilized in the associated heat port definition.

\section{Electric Machine Models}

In the FundamentalWave library five different induction machine models are provided. Each of these models extends from the partial machine model which is depicted in Fig. 10. Each machine model consists of electrical, mechanical and thermal connectors. The stator windings are accessible by a positive and negative multi phase plug with $m=3$ phases, respresenting the begin and the end of the three stator windings, respectively. Each model that dissipates losses is equipped with a thermal heat port. Each of these thermal heat ports is connected to an internal thermal port (super connector). In order to consider heat flow and temperature exchange with external thermal models an optional external thermal port is provided. Dependent on a boolean parameter useThermalport either the external thermal port is used or the losses are dissipated to an internal constant temperature ambient. The shaft end of the machine is represent by the rotational connector $f$ lange. By default, the stator is fixed and thus the rotational connector support, representing the stator housing, is not accessible. Alternatively, if the parameter usesupport is set to true, the rotational connector of the stator housing can be connected with an external mounting model.

The electrical plugs are connected with a model of the stray load losses and a symmetrical model of the stator winding (Fig. 7). The stray load loss model senses the

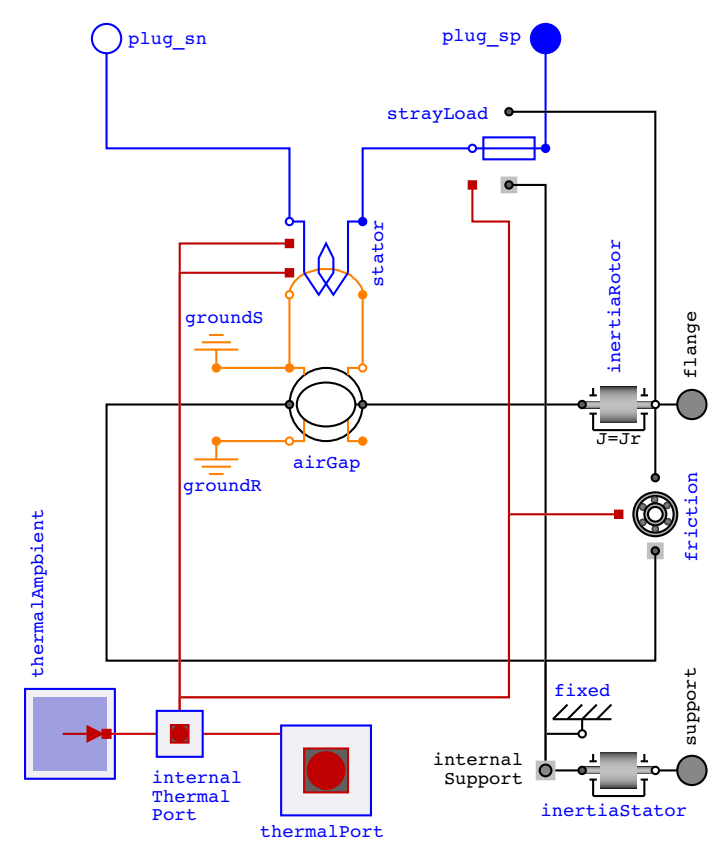

Figure 10: Partial basic induction machine model

actual current and generates mechanical losses proportional to the current and angular velocity. The magnetic ports of the stator winding model are directly connected to stator ports of the air gap model. The inertias of the stator and rotor, respectively, are connected to all the stator and rotor specific rotational connectors of the air gap, stray load loss and friction model, respectively.

The machine parameters of the Machines and the Fundamentalwave library are identical except for one parameter, the effective number of stator turns,effectivestatorTurns, which is solely needed in the FundamentalWave library. This parameter does not affect the operational behavior of the machine but scales the magnetic potential difference and magnetic flux. The fundamental wave connector quantities can therefore only represent the actual physical quantities of the machine, if the real effective number of turns is provided.

\subsection{Asynchronous Induction Machines}

A squirrel cage and slip ring induction machine model are provided in the FundamentalWave library. In the squirrel cage model the magnetic rotor ports of the air gap model are connected with a symmetrical cage model as depicted in Fig. 11. The slip ring induction machine models has a regular three phase winding in the rotor, see Fig. 12. In the current implementation, the rotor core losses are not taken into account. 


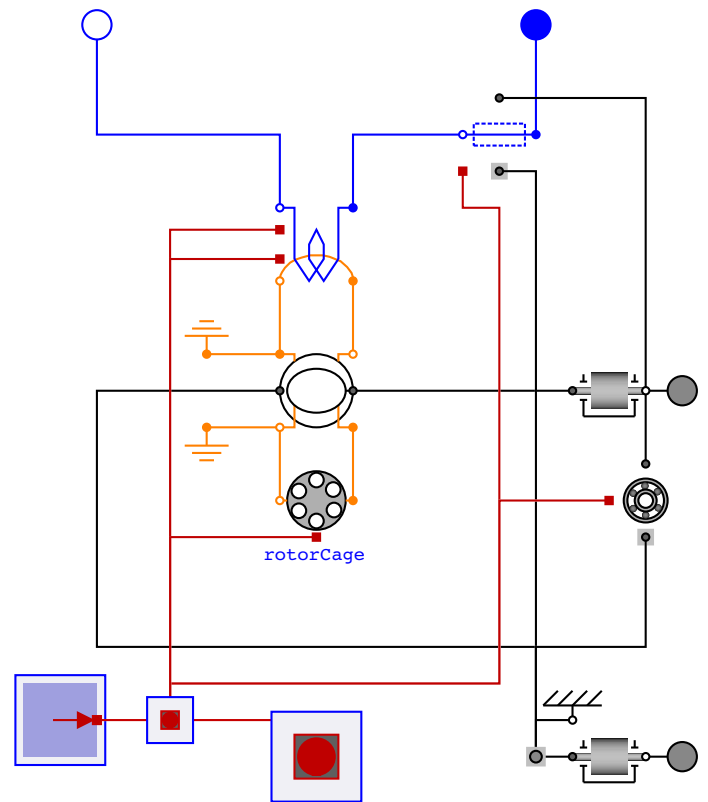

Figure 11: Asynchronous machine with squirrel cage rotor

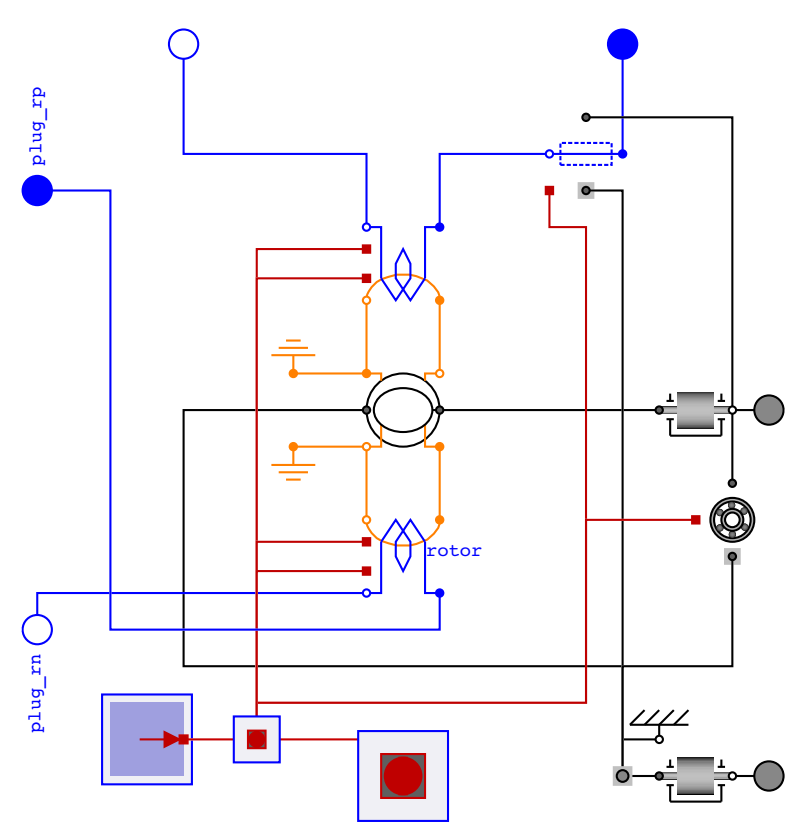

Figure 12: Asynchronous machine with slip ring rotor

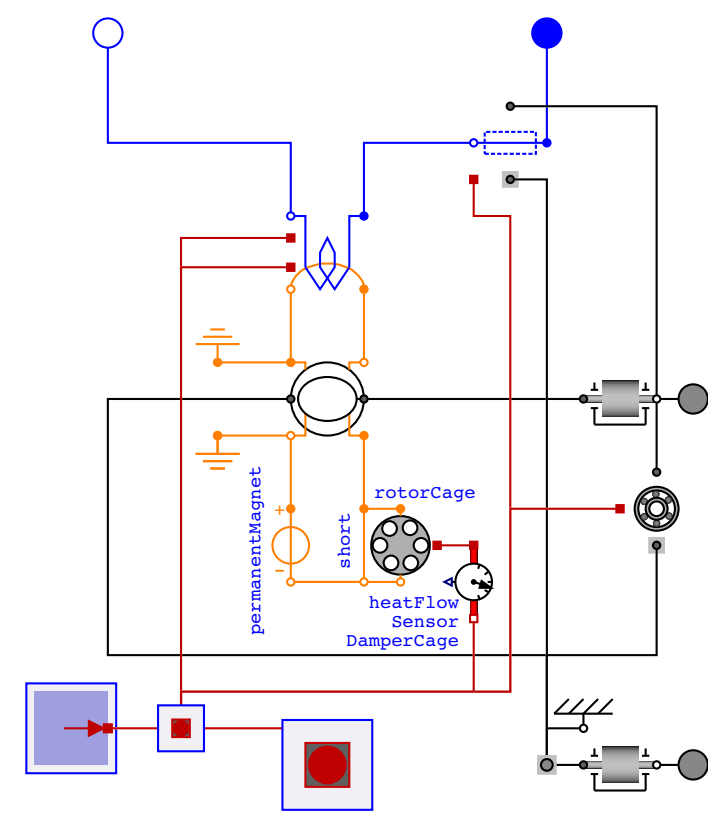

Figure 13: Synchronous machine with permanent magnet

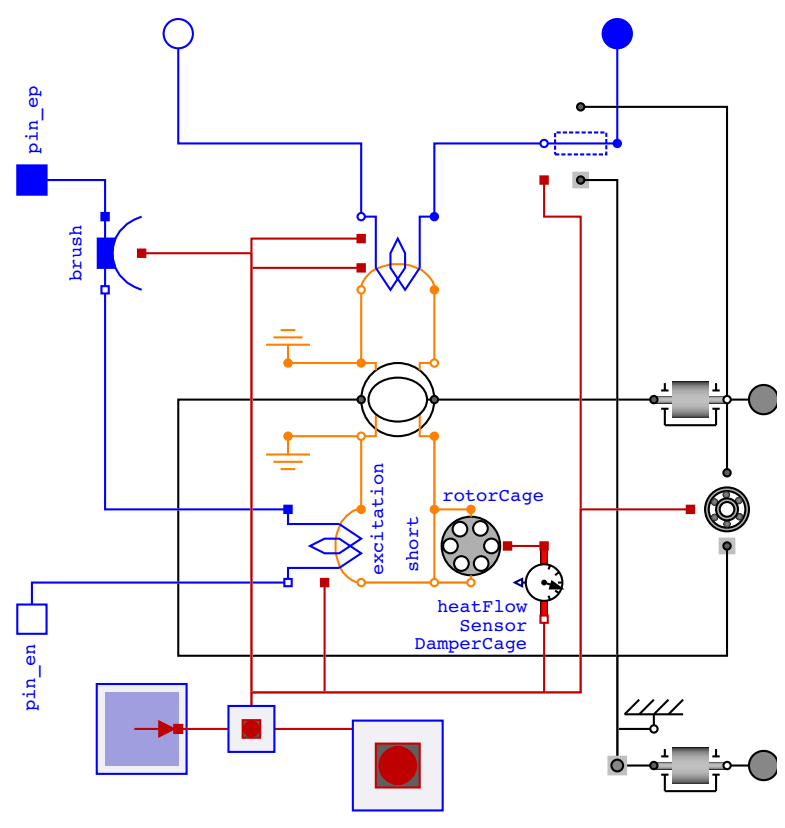

Figure 14: Synchronous machine with electrical excitation 


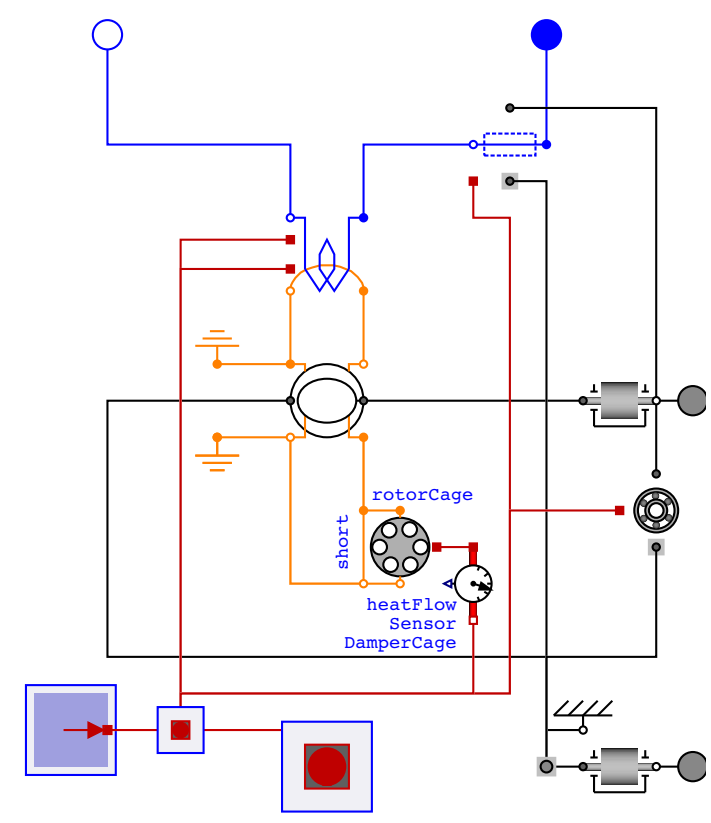

Figure 15: Synchronous machine with reluctance rotor

\subsection{Synchronous Induction Machines}

Three synchronous machine models with electrical excitation, permanent magnet excitation and without excitation but a reluctance rotor are available in the Fundamentalwave library. Each of these machine models is equipped with an optional damper cage. The damper cage parameters include different rotor resistance and rotor leakage inductance parameters in the direct $(d)$ and quadrature $(q)$ axis. If the damper cage is disabled, a magnetic short is connected instead of the damper cage, as show in Fig. 13-15. In order to handle the cage temperature of the super connector in case of the disabled damper cage, a heat flow sensor is uses which can set its connector temperature for consistency reasons. The permanent magnet synchronous machine model depicted in Fig. 13 has a magnetic potential difference source in the rotor representing the permanent magnet. The reluctance of the magnet is inherently represent by the salient main field inductances of the direct $(d)$ and quadrature $(q)$ axis, Lmd and Lmq, respectively. The synchronous machine with electrical excitation has a single phase electrical winding (Fig. 14) and a brush model representing the slip rings of the excitation circuit. In the reluctance synchronous machine model of Fig. 15 no additional excitation is present. The torque generated in this synchronous machine is solely due to rotor saliences, i.e., Lmd $\neq$ Lmq.

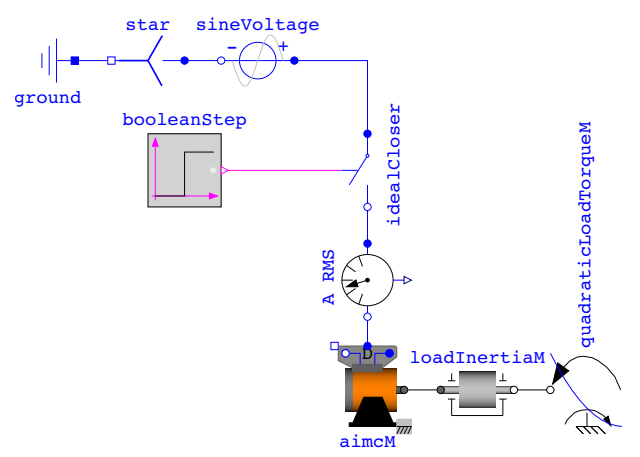

Figure 16: Asynchronous induction machine started direct on line

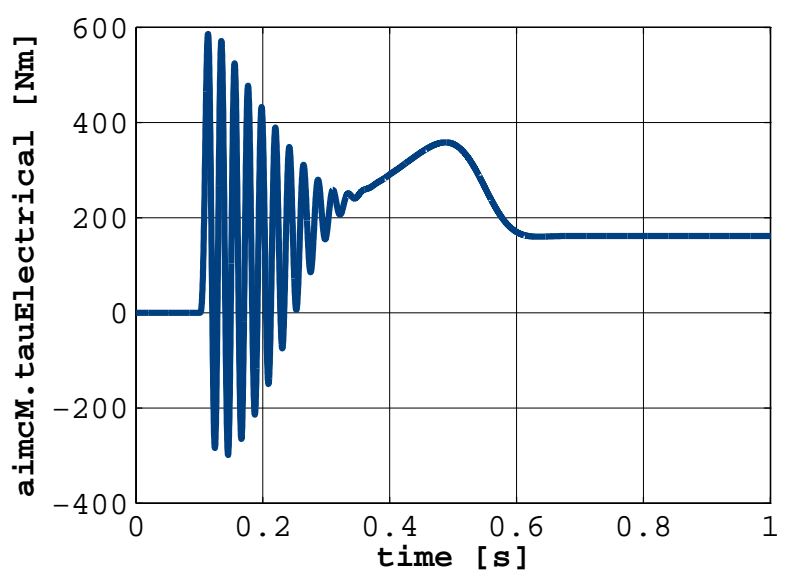

Figure 17: Torque of an asynchronous machine with squirrel cage rotor started directly on line

\subsection{Example}

An example of an asynchronous induction machine started direct on line is presented in Fig. 16, comparable to the included one in the Examples package of the FundamentalWave library. A delta connected induction machine is connected to a stiff voltage supply after closing a switch at $0.1 \mathrm{~s}$. Even if this is not presented here in this paper: the torque developed by the induction machine during starting (Fig. 17 and 18) is exactly matching the results that are obtained by substituting the asynchronous induction machine by a model from the Machines library. So the models are fully compatible with respect to their operational behavior. For the investigated machine the effective number of stator turns was set to 25 which results in the rotor flux results presented in Fig. 19. Immediately after closing the switch at $0.1 \mathrm{~s}$ the rotor frequency is equal to the supply frequency $(50 \mathrm{~Hz})$. After fully accelerating the machine, the slip frequency of the rotor flux is approximately $2 \mathrm{~Hz}$. 


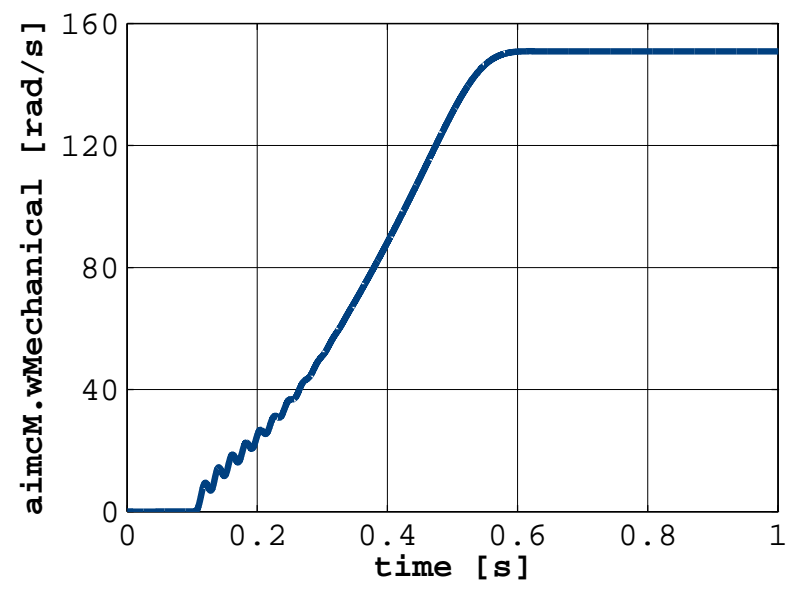

Figure 18: Angular mechanical velocity of an asynchronous machine with squirrel cage rotor started directly on line

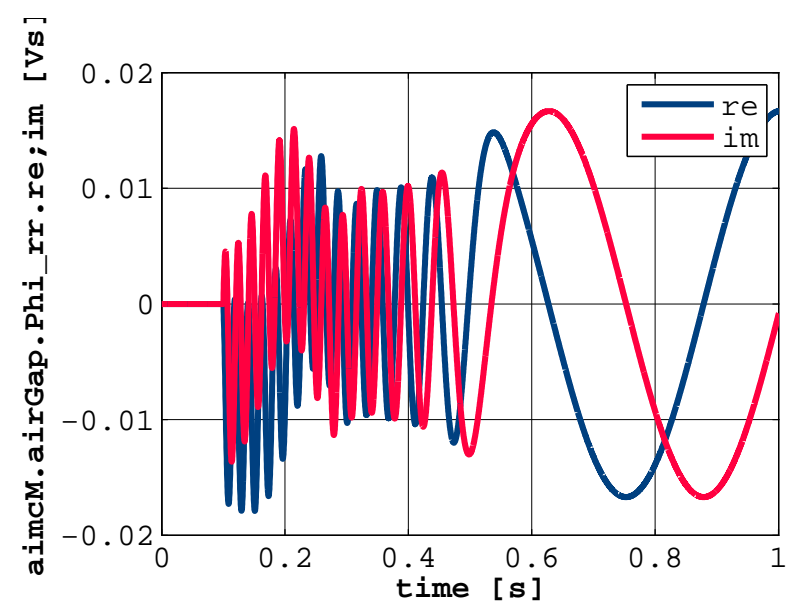

Figure 19: Real and imaginary part of the rotor flux of an asynchronous machine with squirrel cage rotor and 25 stator turns started directly on line

\section{Didactic Aspects and Expandabil- ity}

From a didactic point of view the FundamentalWave electric machines library allows a valuable insight in the concepts of electro mechanical power conversion. Since fundamental wave magnetic domain models are added to the MSL, the magnetic potential and flow variables of an electric machine do have a clear and physical representation. For each component and physical effect in an electrical machine there is a particular model representing this effect. For example, in an induction machine with slip ring rotor the machine basically consists of a stator winding, an air gap and a rotor winding. The total reluctance of the magnetic circuit of the machine is represented by the main field

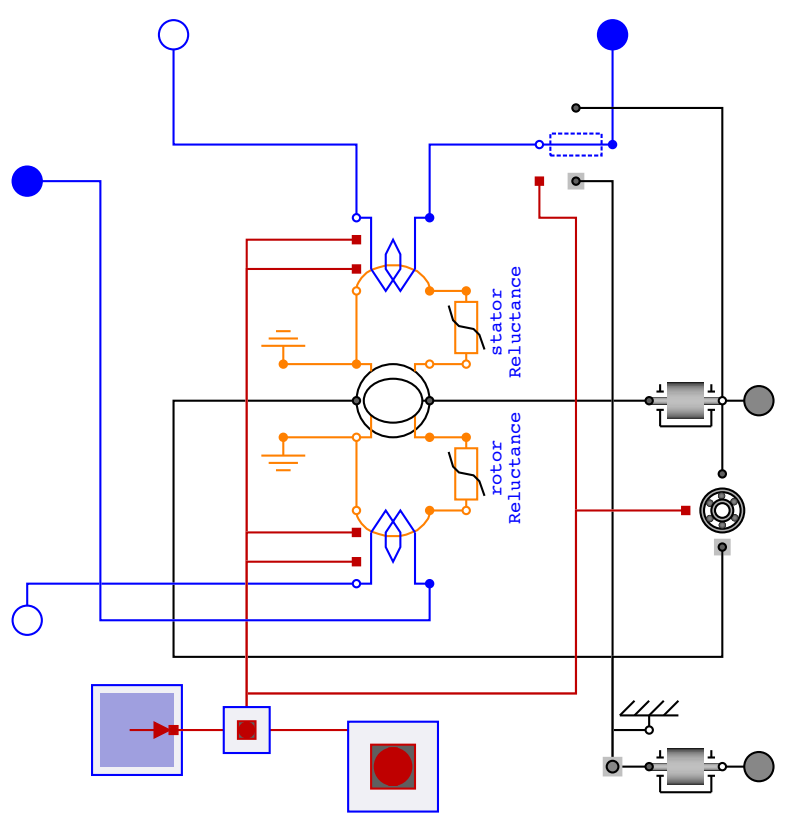

Figure 20: Possible model of an asynchronous induction machine with nonlinear stator and rotor core reluctances

inductance of the air gap model. The reluctances of the stator and rotor teeth and yoke are currently not explicitly modeled.

With the FundamentalWave library the model can certainly be adapted such way that the nonlinear magnetic properties of the stator and rotor core can be considered, as it is proposed in Fig. 20. In such a model the air gap reluctance has to be adapted such way that it only represents the total main field reluctance minus the stator reluctance minus the rotor core reluctance. In such a model the different section of the magnetic main field-stator core, air gap, rotor core-would be represented by the respective magnetic reluctance models.

The temperature dependent ohmic losses of the stator and rotor winding-if present - as well as the stator core losses are inherently taken into account in the winding models. Friction and stray load losses are considered by separate losses. If either of these losses should be modeled in more sophisticated way, e.g., extending the core losses by hysteresis losses, then only the respective loss model has to be replaced and adapted due to the clearly object oriented structure of the components applied in the machine models.

Additional physical effects such as the deep bar effect can be easily modeled by replacing the respective cage model by a more advanced one. In the same way, the entire topology of an asymmetric squirrel cage could be modeled by replacing the three phase symmetrical 
topology by a more sophisticated one [9].

\section{Conclusions}

This paper gives an overview of the FundamentalWave library which is included in the Modelica Standard Library 3.2. The basic idea of the complex magnetic potential difference and the complex magnetic flux is introduced and the basic components and equations are presented. After discussing the machine specific components the common partial machine model and the different asynchronous and synchronous induction machines are presented. From a modeling point of view the basic structure of the fundamental wave electric machine models is very clear and plausible. Each model represents a distinct physically effect and can thus easily be replaced by a more sophisticated model in order to consider additional physical effects.

\section{References}

[1] A. Haumer, C. Kral, H. Kapeller, T. Bäuml, and J. V. Gragger, "The AdvancedMachines library: Loss models for electric machines", Proceedings of the 7th Modelica Conference, pp. 847-854, 2009.

[2] G. J. Retter, Matrix and Space-Phasor Theory of Electrical Machines, Akademiai Kiado, Budapest, 1987.

[3] C. Kral, "Derivation of the space phasor equations and the required parameters of a squirrel cage induction machine with faulty rotor bars", Conference Proceedings of the International Symposium on Diagnostics for Electrical Machines, Power Electronics and Drives, SDEMPED, pp. 395-400, 1999.

[4] J. Stepina, "Raumzeiger in Matrizendarstellung in der Theorie der elektr. Maschine", Archiv fuer Elektrotechnik, vol. 58, pp. 91-97, 1976.

[5] H. Kleinrath, Stromrichtergespeiste Drehfeldmaschinen, Springer Verlag, Wien, 1980.

[6] C. Kral, R. Sprangers, J. Waarma, A. Haumer, O. Winter, and E. Lomonova, "Modeling demagnetization effects in permanent magnet synchronous machines", Proceeding of the XIX International Conference on Electrical Machines 2010, 2010.
[7] M. Beuschel, "A uniform approach for modelling electrical machines", Modelica workshop, pp. 101-108, 2000.

[8] H. Späth, Elektrische Maschinen, Eine Einführung in die Theorie des Betriebsverhaltens, Springer Verlag, Berlin - Heidelberg - New York, 1973.

[9] C. Kral, A. Haumer, and F. Pirker, "A modelica library for the simulation of electrical asymmetries in multiphase machines - the extended machines library", IEEE International Symposium on Diagnostics for Electric Machines, Power Electronics and Drives, The 6th, SDEMPED 2007, Cracow, Poland, pp. 255-260, 2007. 